\title{
Outcome measures of disease activity in rare autoimmune rheumatic diseases
}

\section{AUTHORS}

Dr Alexis Jones, Senior Clinical Research Fellow, Department of Rheumatology, University College London Hospital NHS Trust, London

Ms Alice Cotton Clinical Research Nurse, Department of Rheumatology, University College London Hospital NHS Trust, London

Mrs Jesusa Guinto Clinical Research Nurse, Department of Rheumatology, University College London Hospital NHS Trust, London

Mr James Wilton Clinical Trial Coordinator, Department of Rheumatology, University College London Hospital NHS Trust, London

Dr Coziana Ciurtin Consultant, Department of Rheumatology, University College London Hospital NHS Trust, London NW1 2PQ 


\section{$\underline{\text { ABSTRACT }}$}

Systemic lupus erythematosis (SLE), scleroderma, myositis and Sjögren's sydrome (SS) are rare, complex, multi-systemic rheumatic diseases associated with significant morbidity and mortality. Thorough assessments of disease activity are required to guide clinical management and assess response to new therapies in clinical trials. In this article, we shall review the commonly used outcome measures to assess this group of diseases and discuss the limitations of their use.

\section{INTRODUCTION}

Outcome measures form a crucial component of clinical practice and research. A standardised assessment of disease activity provides an accurate trend of disease activity overtime and prompts changes in patient's management. Over the last decade, there has been burgeoning research into the pathogenesis of SLE, scleroderma, autoimmune myopathies and SS, leading to the development of novel therapeutic targets. Robust measures of disease activity are required to accurately assess the efficacy of these new therapies in clinical trials. The Outcomes Measures in Rheumatologic Trials group (OMERACT) (Tugwell et al., 2007) have established clear constructs for outcome measures; stating that they should be valid, sensitive, reproducible, sensitive to change and feasible. In this article, we shall review the common outcome measures used to assess this group of diseases and the limitations of their use. 


\section{Systemic lupus erythematosus (SLE)}

SLE is a chronic multisystem autoimmune disease with a heterogeneous pattern of clinical and serological manifestations. Pathogenesis of the disease involves a complex interaction between gene susceptibility, hormonal influences and certain environmental triggers which induce autoantibody production (Rahman and Isenberg, 2008). It has an overall incidence of 4.9-5.5 and prevalence of 72.8-97 in recent UK and US population estimates with a 6-10 fold female predominance (Somers et al., 2014) .

Several tools have been developed to assess disease activity both in clinical practice and as primary endpoints in clinical trials. The primary tools used are the BILAG-2004 developed and validated by the British Isles Lupus Assessment Group (Romero-Diaz, Isenberg and Ramsey-Goldman, 2011), SLEADI-2K (systemic lupus erythematosus disease activity index 2000) (Gladman et al., 2003) and SLICC (systemic lupus international collaborating clinics) (Gladman et al., 1996). Limitations of individual disease activity scores lead to the development of composite indices such as SLE responder index (SRI and SRI-50) (Mikdashi and Nived, 2015) (Castrejon et al., 2014) and the BILAG based composite lupus assessment (BLICA) (Castrejon et al., 2014). The most commonly used patient reported outcome score is the Lupus Quality of Life Questionnaire (Lupus-QoL) (Holloway et al., 2014). 


\section{Figure 1: Outcome measures in SLE}

\section{Disease activity scores}

British Isles Lupus Assessment Group Index (BILAG-2004) (Romero-Diaz, Isenberg and Ramsey-

Goldman, 2011)

- Nine systems are measured: constitutional, mucocutaneous, neuropsychiatric, musculoskeletal, cardiorespiratory, gastrointestinal, ophthalmic, renal and haematological

- Features graded as new, the same, worse or improving.

- Incorporates severity and provides assessment scales for individual organs and systems

- Accurate scoring requires that the physician only counts activity that is attributable to lupus

- Activity in each organ system is scored as: $A=$ most active disease (12 points); $B=$ intermediate activity ( 8 points); $C=$ mild, stable disease ( 1 point); $D=$ previous involvement, currently inactive ( 0 points); $E=$ no previous activity ( 0 points). Flares can also be assessed with a severe flare $=A$; new appearance and moderate flare $=B$; and recurrence as score of $D$ or $\mathrm{E}$

Systemic Lupus Erythematosus Disease Activity Index 2000 (SLEADI-2K) (Gladman et al., 2003)

- Global index of disease activity in SLE

- Consists of 24 questions

- Records descriptors of disease activity as present or absent in the preceding 10 or 30 days along with persistent rash, alopecia, oral ulcers and proteinuria limiting its use in clinical trials

- Measures disease activity in 9 organ systems: neurological, musculoskeletal, renal, mucocutaneous, general, cardiac, respiratory, vascular, and hematological with a scoring range of $1-8$

- Provides a single summary score for disease activity with a maximum score of 105

SLE responder index (SRI) (Castrejon et al., 2014)

- Combination index of SELENA-SLEDAI, BILAG and physician global assessment

- A responder is classified as SELENA-SLEDIA improvement of 4 or more points from baseline

- No new BILAG A or B scores

- No worsening of physician global assessment

SLEDAI-2000 Responder Index 50 (SRI 50) (Mikdashi and Nived, 2015)

- Composed of SLEDAI-2K and generates a numerical score that reflects disease activity over the previous 30 days

- Each descriptor identifies at least a 50\% improvement which generates a score for that descriptor

BILAG-Based Composite Lupus Assessment (BICLA) (Castrejon et al., 2014)

- Combination of BILAG, PGA and SLEADI

- Responder classified as no new BILAG $A$ or $B$ scores

- Improvement of BLAG A score to B, BILAG B/C/D to BILAG C/D

- No increase in SLEDAI from baseline

- No worsening of PGA

Damage indices

Systemic Lupus International Collaborating Clinics Damage Index (SLICC) (Gladman et al., 1996)

- Damage assessment index

- Includes 42 items in 12 domains with a maximum score of 46 . Items are rated as being either present or absent with recurring events being scored either 2 or 3.

- Irreversible damage is defined as change in an organ or system that has occurred since the onset of disease and has been present $\geq 6$ months

\section{Patient reported outcomes}

Lupus Quality of Life Questionnaire (LupusQoL) (Holloway et al., 2014)

- Patient reported quality of life questionnaire

- 34 questions covering the preceding four weeks

- Five-point scale ranging from "never" to "all of the time" 


\section{$\underline{\text { Scleroderma }}$}

Scleroderma, also known as systemic sclerosis, is a rare autoimmune disease associated with significant morbidity and mortality. It is characterised by vascular injury and abnormal fibrotic processes that can affect multiple organ systems, including the skin, lungs, gastrointestinal (GI) tract and cardiovascular system.

Skin involvement in scleroderma is almost universal. The modified Rodnan skin score (mRSS) (Clements et al., 1995) is a validated measure of skin disease and has become the most commonly used measure of disease activity in patients with systemic sclerosis. The mRSS correlates with patient derived measures of disease, physical function and mortality. However, there is a high inter-observer variation in this score. Disease of the GI system occurs in approximately $90 \%$ of patients with scleroderma and has a major impact on their health-related quality of life. However, few instruments have been validated for the assessment of GI tract in scleroderma. Interstitial lung disease and pulmonary arterial hypertension are the leading cause of death in patients with scleroderma. Lung function tests and the 6 minute walk test are surrogate markers for these disease parameters in clinical trials. The 6 minute walk test measures the distance a patient can walk in six minutes and has been successfully incorporated into trials of scleroderma-related PAH (Badesch et al., 2000). Raynaud's phenomenon (RP) occurs in more than $90 \%$ of patients with scleroderma and is measured using the RP score (Merkel et al., 2002). Severe Raynaud's can lead to digital ulcers (DU). These are manually counted to provide a DU score. The HAQ I is a widely used patient reported outcome score used in rheumatic diseases (Fries et al., 1980) and has been validated in scleroderma. The scleroderma $H A Q$ is a variation of the HAQ incorporating questions specific to scleroderma disease (Steen and Medsger, 1997). 


\section{Figure 2: Outcome measures in scleroderma}

\section{Disease activity scores \\ Modified Rodnan skin score (mRSS) (Clements et al., 1995)}

Skin thickness across 17 regions of the body. Clinician uses index finger and thumb to roll or gently pinch skin. A scale 0-3 is applied. 0-No thickening, 1-Mild thickening, 2-Moderate thickening, 3-Severe thickening Pulmonary function tests

Vital capacity (VC), Forced vital capacity and Diffusing capacity for carbon monoxide are important variables in the assessment of lung involvement

6 minute walk test

The 6-minute walk test (6MWT) measures the distance a person can walk in 6 minutes

Raynaud's condition score (Merkel et al., 2002)

The RCS is calculated from a summation of 1- or 2-week daily patient self-assessments of RP activity using a 0 to 10 ordinal scale. The RCS incorporates the cumulative daily frequency, duration, severity, and impact of RP attacks.

Digital ulceration count

Manual count of the number of digital ulcers

\section{Patient reported outcomes}

HAQ-DI (Fries et al., 1980)

The HAQ-DI assesses eight disability categories over the past 7 days (dressing/grooming, arising, eating, walking, hygiene, reach, grip, common daily activities). Items are rated on a 4-point scale, ranging from 0 (without any difficulty) to 3 (unable to do), with higher scores indicating greater functional disability. The total score is the mean of the highest scores of each of the eight categories, ranging from 0 (no disability) to 3 (severe disability).

The Scleroderma HAQ (SHAQ) (Steen and Medsger, 1997)

Includes the disability and pain scales of the HAQ plus five visual analogue scales (VASs) that patients use to rate scleroderma-specific problems in the preceding week including pulmonary disease, digital ulcers, Raynaud's phenomenon, GI disease, and skin disease

\section{Inflammatory myopathies}

The idiopathic inflammatory myopathies (IIM) are characterised by auto immune mediated

muscle inflammation and weakness. They have a worldwide prevalence of 14 in 100,000

(Meyer et al., 2015). Adult polymyositis (PM), dermatomyositis (DM), and juvenile

dermatomyositis (JDM) are among the most frequent of the IIM. During the past decade, collaborations such as the International Myositis Assessment \& Clinical Studies Group

(IMACS) have undertaken projects to define core measures of disease activity and damage in myositis and dermatomyositis, and to develop and validate tools for these measures

(Isenberg et al., 2004). 
The most commonly-used tools include the Manual Muscle Test 8 (MMT8) (Miller et al., 2001); Myositis Intention to Treat (MITAX); and Myositis Disease Activity Assessment Tool (MYOACT) (Isenberg et al., 2004). Disease damage measures are used to assess the persistent change in anatomy, physiology, pathology or function resulting from previously active disease or complications of therapy. Usually, changes are post-inflammatory, cumulative and irreversible. Damage should be present for at least six months despite previous immunotherapy, rehabilitation or other therapy. The most commonly-used tool to assess disease damage is Myositis Damage Index (MDI) (Isenberg et al., 2004).

In addition to measuring myositis-specific activity and damage, Rider and colleagues (2011) also recommend the use of the following tools: general tools of global disease activity (e.g. physician and patient visual analogue scales - VAS); functional assessment tools (e.g. Health Activity Questionnaire - HAQ, and childhood myositis assessment score); and patientreported outcome measures (e.g. health-related quality of life measures such as SF36 for adults or CHQ-PF50 for children). 


\section{Figure 3: Outcomes measures in myositis}

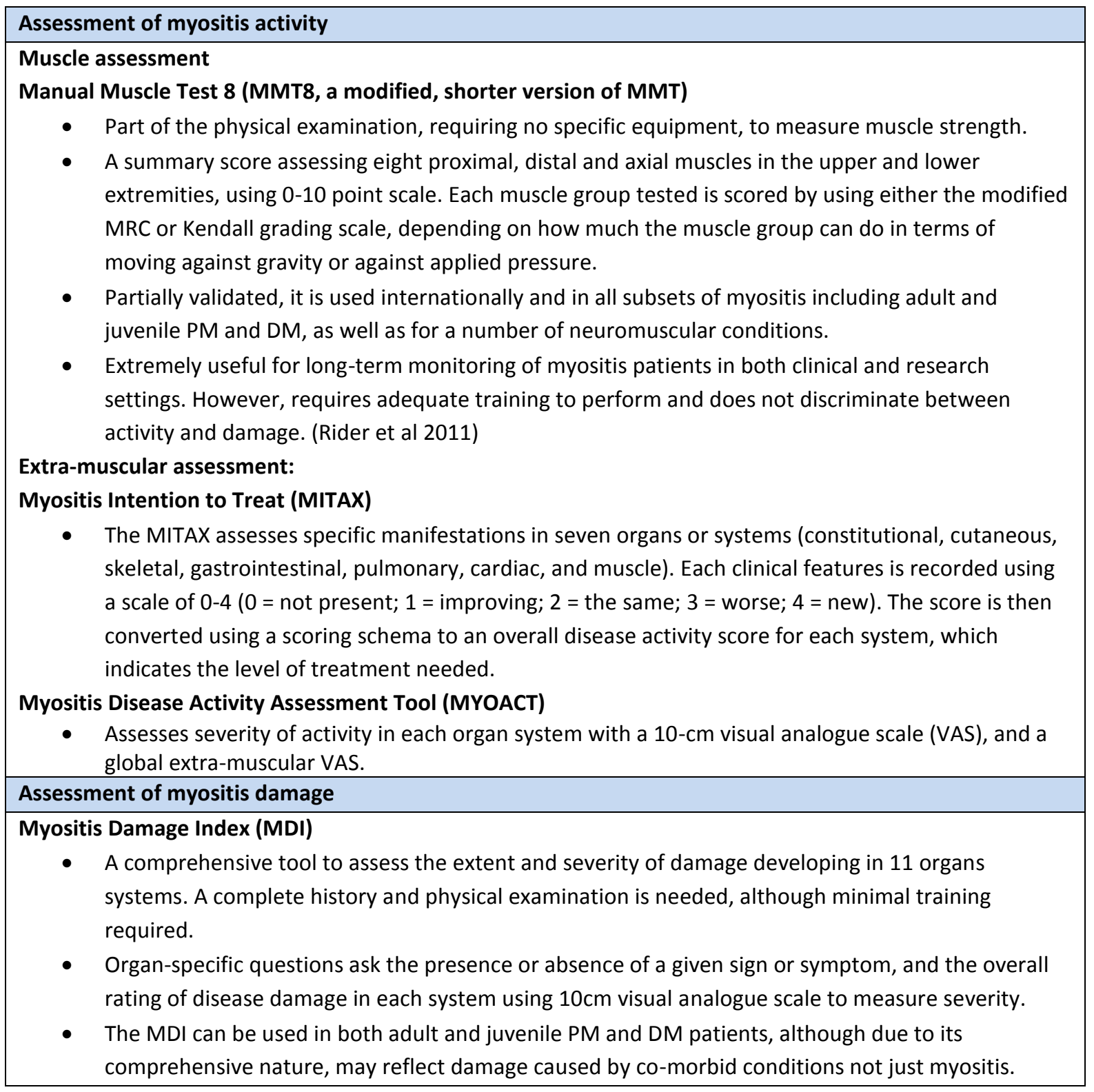

\section{Dermatomyositis}

Myositis occurring with characteristic skin and nail manifestations is coined

dermatomyositis (DM). These manifestations may include Gottron's papules, helitrope rash, photo-distributed erythema, poikiloderma, dilated nail fold capillaries, scalp involvement and calcinosis cutis. The Cutaneous Dermatomyositis Disease Area and Severity Index (CDASI), is the most commonly used combined tool in clinical practice and therapeutic studies for DM (Klein et al., 2008). It is a clinician scored instrument that measures skin 
activity and damage. Activity is measured in three areas - erythema, scale, and erosion/ulceration. Damage is measured in two areas - poikiloderma and calcinosis. In addition, Gottrons papules, periungual changes and alopecia are also scored.

\section{Figure 4: Dermatomyositis-related rash}

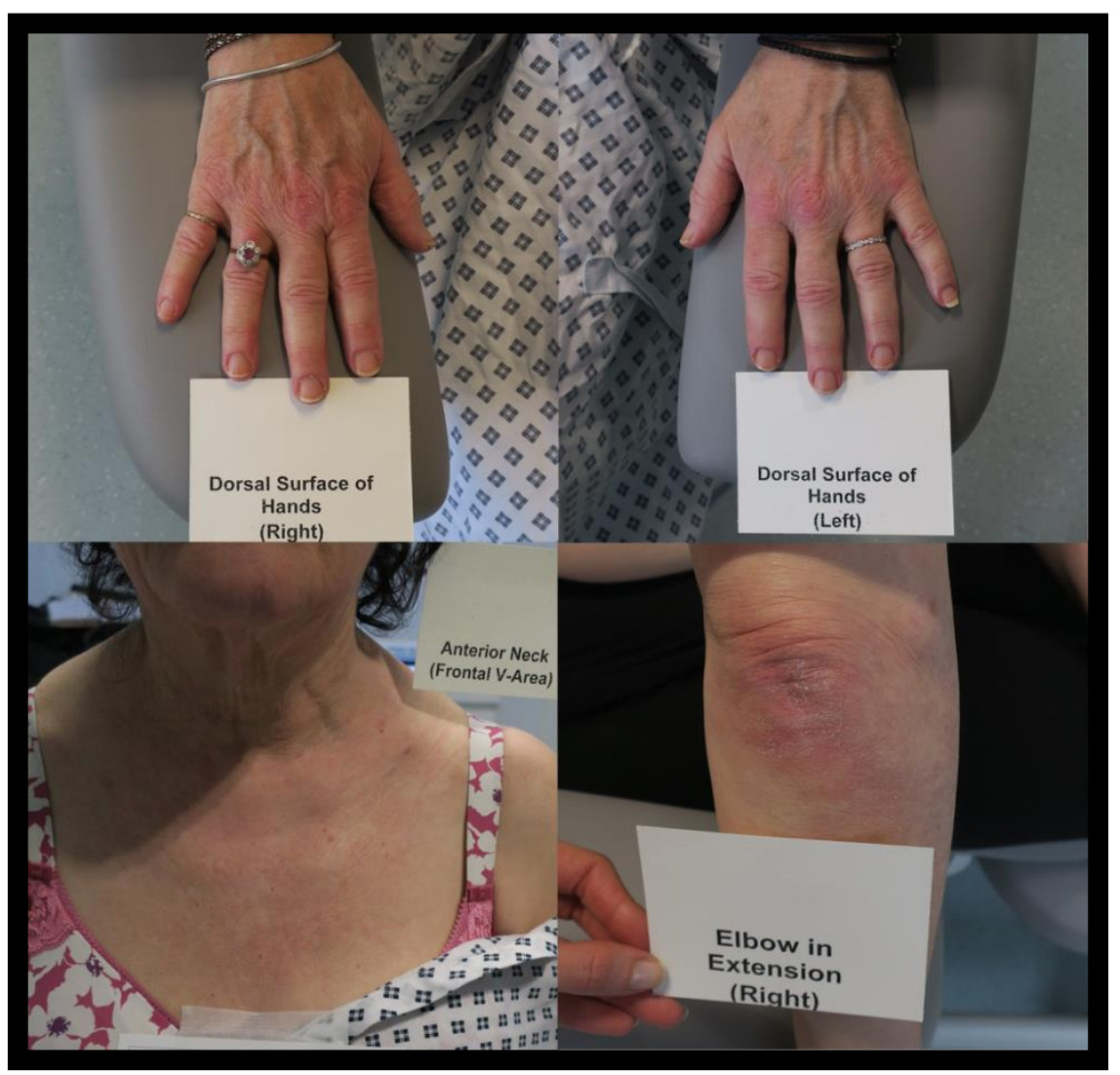


Figure 5: Example of CDASI scoring of dermatomyositis rash

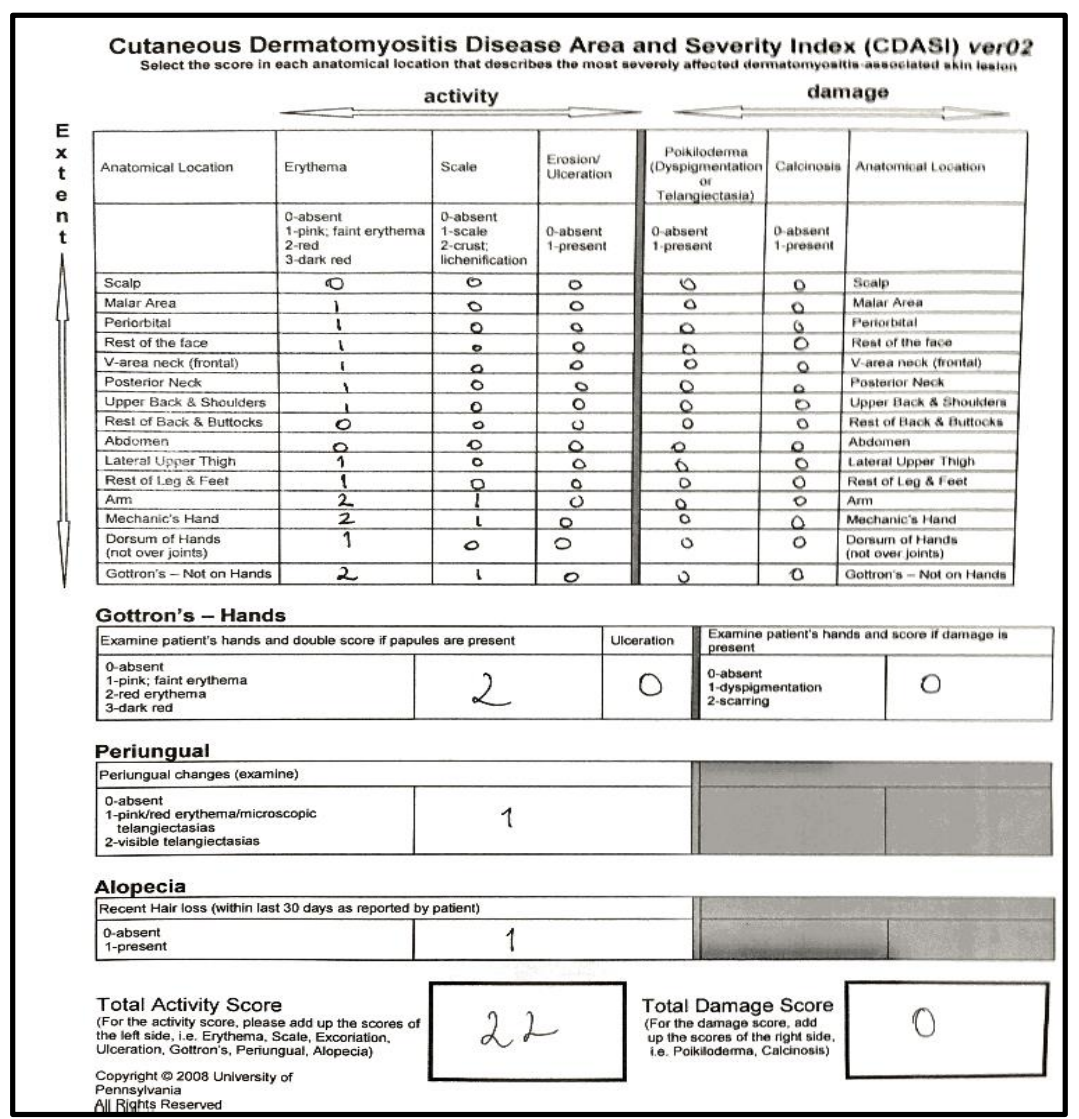

\section{Sjögren's sydrome}

Sjögren's syndrome (SS) is a chronic autoimmune disorder affecting approximately $0.1-0.4 \%$ of the general population with a female-to-male ratio of $9: 1$, usually diagnosed in the fourth and fifth decades of life (Daridon et al., 2007). Clinically, SS is characterised by ocular and oral dryness developed because of the autoimmune infiltrating process affecting the exocrine glands. It may occur either alone, as primary SS, or in association with other autoimmune disease, often rheumatoid arthritis, systemic lupus erythematosus or systemic sclerosis, in which case is called secondary SS. Clinical, laboratory and histological features can be used to classify the systemic manifestations of SS as periepithelial or tissue-specific 
(including liver, lung and kidney) and extraepithelial (including vasculitis, peripheral neuropathy, renal involvement and myositis) (Fox et al., 1984).

In the past decades, a core set of domains was defined to facilitate the complex assessment of SS patients' outcomes (Seror et al., 2012). This included sicca symptoms, objective measurements of tear and saliva production, fatigue, quality of life, disease activity and damage indexes.

Significant efforts have been made to develop valid tools for the assessment of various clinical and laboratory manifestation of SS, as the disease can have a heterogeneous presentation. A large international project supported by EULAR, led to the development of two consensus disease activity indexes: the EULAR Sjögren's Syndrome Patients Reported Index (ESSPRI) (Seror et al., 2011), and the EULAR Sjögren's Syndrome Disease Activity Index (ESSDAI) (Seror et al., 2015), a systemic activity index to assess systemic manifestations, which are the most used outcome measures in SS. In addition, patient questionnaires such as the Profile of Fatigue and Discomfort (PROFAD) and Sicca Symptoms Inventory (SSI) have also been developed. The table below details the most used disease specific outcome measures in SS. 


\section{Figure 6: Outcomes measures in Sjögren's sydrome}

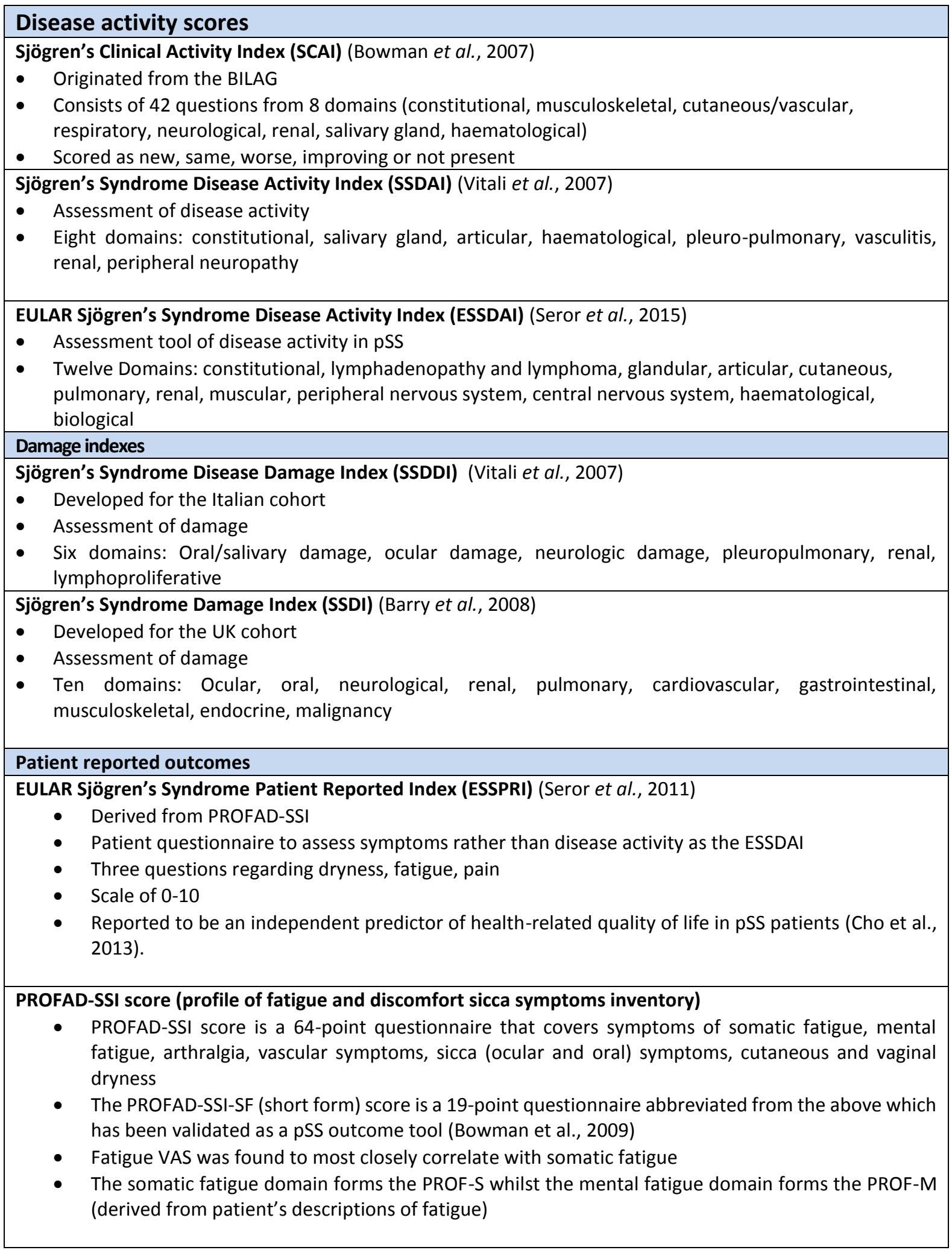


The ESSDAI and ESSPRI scores are currently used as gold standard in clinical trials. An ESSDAI $\geq 5$ signifies moderately active disease, while a minimal clinically important improvement is defined as a decrease of at least 3 points (Seror et al., 2011). An ESSPRI score above 5 defines significant impact of SS associated symptoms on patients' quality of life (Cho et al., 2013). Both ESSDAI and ESSPRI are found to be sensitive to change (Meiners et al., 2012) ; therefore they are the most used outcome measures in SS.

\section{Conclusions}

A number of outcome measures have been validated for the assessment of rare rheumatologic diseases. The multi-systemic nature of these diseases pose a significant challenge to accurately capture the spectrum of disease activity and damage. Furthermore, the rarity of these diseases limits the power of validity and reproducibility assessments for outcome measures. As we advance our understanding of the pathogenesis of these diseases, and develop novel therapeutic targets, refinement of these outcome measures will become necessary. 


\section{REFERENCES}

Badesch, D. B. et al. (2000) 'Continuous intravenous epoprostenol for pulmonary hypertension due to the scleroderma spectrum of disease. A randomized, controlled trial.', Annals of internal medicine, 132(6), pp. 425-34. doi: 10.1016/0002-8223(94)92210-1.

Barry, R. J. et al. (2008) 'The Sjogren's Syndrome Damage Index--a damage index for use in clinical trials and observational studies in primary Sjogren's syndrome.', Rheumatology (Oxford, England), 47(8), pp. 1193-1198. doi: 10.1093/rheumatology/ken164.

Bowman, S. J. et al. (2007) 'Sjogren's Systemic Clinical Activity Index (SCAI)--a systemic disease activity measure for use in clinical trials in primary Sjogren's syndrome', Rheumatology (Oxford), 46(12), pp. 1845-1851. doi: 10.1093/rheumatology/kem280.

Castrejon, l. et al. (2014) 'Indices to assess patients with systemic lupus erythematosus in clinical trials, long-term observational studies, and clinical care', Clinical \& Experimental Rheumatology, 32, p. S-85-95. Available at:

http://ovidsp.ovid.com/ovidweb.cgi?T=JS\&CSC=Y\&NEWS=N\&PAGE=fulltext\&D=medI\&AN=2 5365095

http://oxfordsfx.hosted.exlibrisgroup.com/oxford?sid=OVID:medline\&id=pmid:25365095\&i $\mathrm{d}=\mathrm{doi}: \& i s s n=0392-856 \mathrm{X} \& \mathrm{isbn}=\&$ volume=32\&issue=5\&spage=S\&pages=S-8595\&date $=2014 \&$ title $=$.

Cho, H. J. et al. (2013) 'The EULAR Sjogren's syndrome patient reported index as an independent determinant of health-related quality of life in primary Sjogren's syndrome patients: in comparison with non-Sjogren's sicca patients.', Rheumatology (Oxford, England), 52(12), pp. 2208-17. doi: 10.1093/rheumatology/ket270.

Clements, P. et al. (1995) 'Inter and intraobserver variability of total skin thickness score (Modified Rodnan TSS) in systemic sclerosis', Journal of Rheumatology, 22(7), pp. 12811285.

Daridon, C. et al. (2007) 'Aberrant expression of BAFF by B lymphocytes infiltrating the salivary glands of patients with primary Sjögren's syndrome', Arthritis and Rheumatism, 56(4), pp. 1134-1144. doi: 10.1002/art.22458.

Fox, R. I. et al. (1984) 'Primary sjogren syndrome: Clinical and immunopathologic features', Seminars in Arthritis and Rheumatism, 14(2), pp. 77-105. doi: 10.1016/00490172(84)90001-5.

Fries, J. F. et al. (1980) 'Measurement of patient outcome in arthritis', Arthritis \& Rheumatism, 23(2), pp. 137-145. doi: 10.1002/art.1780230202.

Gladman, D. et al. (1996) 'Systemic lupus International Collaborating Clinics Conference on assessment of lupus flare and quality of life measures in SLE. San Francisco, USA, October 22, 1995', in Journal of Rheumatology, pp. 1953-1955.

Gladman, D. D. et al. (2003) 'Accrual of organ damage over time in patients with systemic lupus erythematosus.', The Journal of rheumatology, 30(9), pp. 1955-1959.

Holloway, L. et al. (2014) 'Patient-reported outcome measures for systemic lupus erythematosus clinical trials: A review of content validity, face validity and psychometric 
performance', Health and Quality of Life Outcomes, 12(1). doi: 10.1186/s12955-014-0116-1.

Isenberg, D. A. et al. (2004) 'International consensus outcome measures for patients with idiopathic inflammatory myopathies. Development and initial validation of myositis activity and damage indices in patients with adult onset disease', Rheumatology, 43(1), pp. 49-54. doi: $10.1093 /$ rheumatology/keg427.

Klein, R. Q. et al. (2008) 'Comparison of the reliability and validity of outcome instruments for cutaneous dermatomyositis', British Journal of Dermatology, 159(4), pp. 887-894. doi: 10.1111/j.1365-2133.2008.08711.x.

Meiners, P. M. et al. (2012) 'Responsiveness of disease activity indices ESSPRI and ESSDAI in patients with primary Sjogren's syndrome treated with rituximab', Annals of the Rheumatic Diseases, 71(8), pp. 1297-1302. doi: 10.1136/annrheumdis-2011-200460.

Merkel, P. A. et al. (2002) 'Measuring disease activity and functional status in patients with scleroderma and Raynaud's phenomenon', Arthritis and Rheumatism, 46(9), pp. 2410-2420. doi: 10.1002/art.10486.

Meyer, A. et al. (2015) 'Incidence and prevalence of inflammatory myopathies: a systematic review.', Rheumatology (Oxford, England), 54(1), pp. 50-63. doi:

10.1093/rheumatology/keu289.

Mikdashi, J. and Nived, O. (2015) 'Measuring disease activity in adults with systemic lupus erythematosus: The challenges of administrative burden and responsiveness to patient concerns in clinical research', Arthritis Research and Therapy. doi: 10.1186/s13075-0150702-6.

Miller, F. W. et al. (2001) 'Proposed preliminary core set measures for disease outcome assessment in adult and juvenile idiopathic inflammatory myopathies.', Rheumatology, 40(11), pp. 1262-1273. doi: 10.1093/rheumatology/40.11.1262.

Rahman, A. and Isenberg, D. A. (2008) 'Systemic lupus erythematosus.', The New England journal of medicine, 358(9), pp. 929-39. doi: 10.1056/NEJMra071297.

Romero-Diaz, J., Isenberg, D. and Ramsey-Goldman, R. (2011) 'Measures of adult systemic lupus erythematosus: Updated Version of British Isles Lupus Assessment Group (BILAG 2004), European Consensus Lupus Activity Measurements (ECLAM), Systemic Lupus Activity Measure, Revised (SLAM-R), Systemic Lupus Activity Questi', Arthritis Care and Research, 63(SUPPL. 11). doi: 10.1002/acr.20572.

Seror, R. et al. (2011) ‘EULAR Sjögren's Syndrome Patient Reported Index (ESSPRI): Development of a consensus patient index for primary Sjögren's syndrome', Annals of the Rheumatic Diseases, 70(6), pp. 968-972. doi: 10.1136/ard.2010.143743.

Seror, R. et al. (2012) 'Outcome measures for primary Sjögren's syndrome', Journal of Autoimmunity, pp. 97-102. doi: 10.1016/j.jaut.2012.01.013.

Seror, R. et al. (2015) 'EULAR Sjögren's syndrome disease activity index (ESSDAI): A user guide', RMD Open, 1(1). doi: 10.1136/rmdopen-2014-000022. 
Somers, E. C. et al. (2014) 'Population-based incidence and prevalence of systemic lupus erythematosus: The Michigan lupus epidemiology and surveillance program', Arthritis and Rheumatology, 66(2), pp. 369-378. doi: 10.1002/art.38238.

Steen, V. D. and Medsger, T. A. (1997) 'The value of the health assessment questionnaire and special patient-generated scales to demonstrate change in systemic sclerosis patients over time', Arthritis \& Rheumatism, 40(11), pp. 1984-1991. doi: 10.1002/art.1780401110.

Tugwell, P. et al. (2007) 'OMERACT: An international initiative to improve outcome measurement in rheumatology', Trials. doi: 10.1186/1745-6215-8-38.

Vitali, C. et al. (2007) 'Sjögren's syndrome disease damage index and disease activity index: Scoring systems for the assessment of disease damage and disease activity in Sjögren's syndrome, derived from an analysis of a cohort of Italian patients', Arthritis and Rheumatism, 56(7), pp. 2223-2231. doi: 10.1002/art.22658. 